\title{
Comorbilidad de los trastornos por consumo de sustancias con otros trastornos psiquiátricos en Centros Residenciales de Ayuda-Mutua para la Atención de las Adicciones
}

\author{
Rodrigo Marín-Navarrete, ${ }^{1}$ Corina Benjet, ${ }^{2}$ Guilherme Borges, ${ }^{2}$ Angélica Eliosa-Hernández, ${ }^{1}$ \\ Ricardo Nanni-Alvarado, ${ }^{3}$ Marcos Ayala-Ledesma, ${ }^{1}$ José Fernández-Mondragón, ${ }^{1}$ María Elena Medina-Mora ${ }^{4}$
}

Artículo original

\section{SUMMARY}

Substance use disorders have a high degree of comorbidity with other psychiatric disorders; it has been reported that the prevalence of comorbidity is higher in psychiatric $(20-50 \%)$ and addiction $(50-75 \%)$ treatment settings than in household or student populations. Because of limited treatment alternatives and greater treatment needs, MutualAid groups have become relevant in the last decades. A modality of Mutual-Aid for addiction treatment that has proliferated in Mexico has taken the form of residential Mutual-Aid centers called "anexos" in Spanish. The objective of this study was to estimate the prevalence of lifetime comorbidity between substance use disorders and psychiatric disorders in those who attended these residential Mutual-Aid centers. The initial sample consisted of 535 male participants diagnosed with a substance use disorder, but only 346 fulfilled the inclusion criteria to continue with the evaluation. Only males were included as the participating centers only admit males. Psychiatric diagnosis was evaluated with the Composite International Diagnostic Interview (WMH-CIDI) using DSM-IV criteria. The results showed that $75.72 \%$ met criteria for any comorbid psychiatric disorder, the most frequent being attention deficit and conduct disorders, followed by anxiety disorders, separation anxiety disorders, mood disorders, impulse control disorders and least frequently eating disorders. While the study is limited by its nonrepresentative sample, the findings provide valuable information for a hidden population for which there is a dearth of information and points to the need for integrative services which address both addiction and comorbid psychiatric disorders simultaneously.

Key words: Addiction, treatment settings, comorbidity, psychiatric disorders.

\section{RESUMEN}

La comorbilidad de los trastornos por consumo de sustancias con otros trastornos mentales presenta una importante prevalencia; se ha reportado que ésta es mayor en los centros de tratamiento psiquiátrico (20$50 \%$ y para las adicciones $(50-75 \%)$ en comparación con la población abierta. Una modalidad de Ayuda-Mutua para la atención de las adicciones común en México es la de los Centros Residenciales y Casas de Recuperación para las adicciones, también llamados "anexos". El objetivo del estudio fue estimar la prevalencia de comorbilidad de los trastornos por consumo de sustancias con otros trastornos psiquiátricos en una muestra de participantes de sexo masculino adscritos a los Centros Residenciales de Ayuda-Mutua para la Atención de las Adicciones (CRAMAA). Se captó a un total de 535 participantes, de los cuales 346 cumplieron los criterios de inclusión y fueron evaluados. La evaluación diagnóstica de los trastornos por uso de sustancias y los 17 trastornos psiquiátricos comórbidos se realizó con la Entrevista Internacional Diagnóstica Compuesta (WMH-CIDI). Los resultados mostraron que $75.72 \%$ cumplía con criterios diagnósticos para algún trastorno psiquiátrico comórbido, siendo los más prevalentes los trastornos por déficit de atención y comportamiento perturbador, seguidos por los trastornos de ansiedad, la ansiedad por separación, los trastornos afectivos, los trastornos por control de impulsos y con menor frecuencia los trastornos de la conducta alimentaria. En la mayoría de los casos $(83.59 \%)$, los trastornos psiquiátricos comórbidos precedieron a los trastornos adictivos. Este estudio constituye una aportación que puede considerarse para futuras propuestas en políticas públicas, que se traduzcan en acciones para ofertar servicios que atiendan las adicciones y los trastornos psiquiátricos de manera integral.

Palabras clave: Adicciones, comorbilidad, trastornos psiquiátricos, centros de atención.

\footnotetext{
Unidad de Ensayos Clínicos en Adicciones y Salud Mental. Subdirección de Investigaciones Clínicas (INPRFM).

2 Dirección de Investigaciones Epidemiológicas y Psicosociales, INPRFM.

Centro Nacional para la Prevención y Control de las Adicciones (CENADIC).

4 Dirección General del INPRFM.

Correspondencia: Rodrigo Marín-Navarrete. Unidad de Ensayos Clínicos, Sub-Dirección de Investigaciones Clínicas, Instituto Nacional de Psiquiatría Ramón de la Fuente Muñiz, Calz. México-Xochimilco 101, 14370, México DF. Tel. (55) 4160-5480 y 5481. E-mail: rmarin@inprf.gob.mx

Recibido primera versión: 14 de diciembre de 2012. Segunda versión: 10 de junio de 2013. Aceptado: 21 de junio de 2013.
} 


\section{INTRODUCCIÓN}

El consumo de sustancias psicoactivas es un fenómeno que ha cobrado relevancia en las últimas décadas, debido al impacto en la calidad de vida del paciente y su familia, así como al costo económico y social que ocasiona a la población. De acuerdo con lo reportado por la literatura científica, los jóvenes y los adultos jóvenes son los más afectados por el uso de drogas, pues se ha identificado que las generaciones actuales se encuentran más expuestas a ellas. ${ }^{1}$

Aunado a lo anterior, la comorbilidad de los trastornos por consumo de sustancias con otros trastornos mentales presenta una importante prevalencia según reporta la bibliografía internacional. ${ }^{2-4}$ Los resultados de estudios realizados en sedes de tratamiento (hospitales psiquiátricos, clínicas de desintoxicación y de tratamiento para las adicciones) muestran mayor comorbilidad aun frente a estudios epidemiológicos realizados en escuelas y hogares (29\%, aproximadamente) ${ }^{5}$ ya que los primeros reportan que la prevalencia de la comorbilidad de trastornos por consumo de sustancias con otros trastornos psiquiátricos oscila entre 50\% y 75\% en participantes cuya sede de tratamiento se especializa en la atención de las adicciones. ${ }^{3,4}$ Asímismo, esta comorbilidad en la población de pacientes cuya sede de tratamiento se especializa en la atención psiquiátrica general oscila entre 20 y 50\%.,6

En los últimos años, se han realizado diversas investigaciones que tienen por objeto estudiar la comorbilidad en poblaciones especiales, entre ellas, indigentes, ${ }^{7}$ reclusos, ${ }^{8,9}$ adolescentes, ${ }^{10}$ mujeres, ${ }^{11}$ pacientes con enfermedad mental grave, ${ }^{12,13}$ unidades de tratamiento residencial y ambulatorio. ${ }^{14-16}$ En ellos se ha encontrado que los trastornos que presentan una mayor comorbilidad con los trastornos por consumo de sustancias son los del estado de ánimo, los de ansiedad, los psicóticos y los de la personalidad antisocial ${ }^{17}$ (cuadro 1).

Es por lo anterior que la importancia de estudiar la comorbilidad de los trastornos por consumo de sustancias con otros trastornos mentales no sólo radica en sus elevados índices de prevalencia, sino en el impacto que ésta tiene en la salud pública. Según lo que reporta la literatura especializada, las personas que presentan esta comorbilidad desarrollan mayor gravedad de la sintomatología adictiva y psiquiátrica, situación que se asocia significativamente en el deterioro de la calidad de vida y del funcionamiento psicosocial del individuo. ${ }^{2-4,18}$

Además de lo ya descrito, existen otras implicaciones a las que estos pacientes se ven expuestos, como el riesgo de padecer alguna enfermedad infectocontagiosa (VIH, hepatitis B y/o C), incremento de la ideación suicida, conducta suicida y suicidio consumado, mayor riesgo de involucrarse en problemas legales y sociales, lo que puede aumentar la probabilidad de indigencia y/o reclusión. ${ }^{2-4,18}$

Aunado a lo anterior, los pacientes con esta comorbilidad presentan falta de adherencia para mantenerse y com-

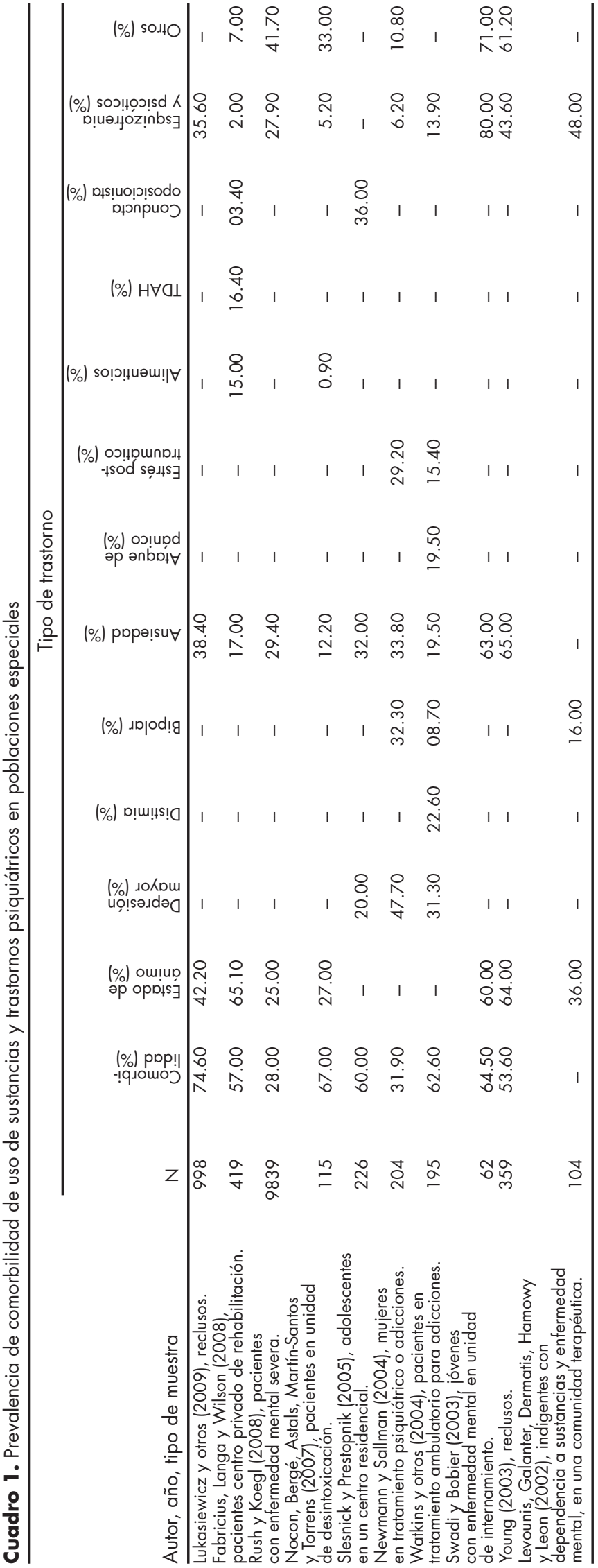


pletar tratamientos psicológicos y/o farmacológicos, lo que conlleva a intentos de atención poco exitosos en función del alto índice de recaída., 3,4,19,20

Por otra parte, uno de los aspectos de relevancia que señala la bibliografía es la dificultad que los pacientes enfrentan al no encontrar programas de tratamiento que integren la atención psiquiátrica, así como la adictológica, recibiendo tratamientos paralelos o secuenciados, lo que disminuye su efectividad e incrementa sus costos. ${ }^{2-4,21}$

En México se cuenta con pocos estudios que tengan por objeto estimar la comorbilidad de trastornos por consumo de sustancias con otros trastornos psiquiátricos o mentales. De ellos uno de los más representativos es el publicado por Caraveo-Anduaga y Colmenares-Bermúdez (2002), mismo que se desarrolló por medio de encuestas en hogares del Distrito Federal.

Según los autores, los resultados reflejaron que en su mayoría el trastorno psiquiátrico precede al consumo de sustancias, por lo que éste podría representar en cierta forma un medio de autorregulación para disminuir la sintomatología del padecimiento mental. ${ }^{22}$ Asimismo, los autores reportaron que los trastornos de ansiedad y los trastornos afectivos fueron los más prevalentes en comorbilidad con los trastornos por consumo de sustancias, y que además la gravedad del consumo incrementa el riesgo de comorbilidad. ${ }^{22}$

Otros estudios hechos en México que han reportado este fenómeno de comorbilidad en centros de tratamiento refieren que el consumo de alcohol entre la población psiquiátrica es elevado, ya que presentan tanto trastornos por abuso como dependencia a esa sustancia. Por ello concluyen que existe una fuerte asociación entre el consumo excesivo de alcohol y otros diagnósticos psiquiátricos, como los trastornos afectivos, de ansiedad, disfunción sexual y esquizofrenia. ${ }^{23,24}$ Sin embargo, estos reportes tienen ya dos décadas y se desconoce la situación actual.

A nivel nacional, debido a las reducidas ofertas profesionales del sector público y lo costosas e inaccesibles que son las ofertas del ámbito privado para la atención de las adicciones, los Centros Residenciales de Ayuda-Mutua para Atención de las Adicciones (CRAMAA) también llamados "anexos", se han convertido en una opción accesible para familiares y pacientes que sufren de trastornos relacionados con el consumo de alcohol y drogas. ${ }^{25}$

La principal característica de estos centros es su heterogeneidad, ya que la mayoría de ellos ofrece una diversidad de servicios residenciales que varían en duración de tres semanas hasta doce meses, dependiendo de la meta o el periodo que establezcan los encargados (servidores) del centro, por lo que en muchos casos el internamiento es solicitado por los familiares y se da en forma involuntaria para el consumidor de sustancias. ${ }^{25}$

Otra característica es su infraestructura, es decir, algunos cuentan con instalaciones amplias, mientras que otras tienen espacios limitados y por tal motivo se presenta haci- namiento en su población. Además es importante mencionar que la estructura jerárquica de estos centros se encuentra constituida por individuos que han logrado mantenerse sin consumo por mayor tiempo y buscan compartir su experiencia induciendo la recuperación en otros. Sin embargo, gran parte de dichos centros no cuenta con el apoyo de especialistas o profesionales de la salud. ${ }^{25,26}$

En función de lo ya descrito, es evidente que existe un vacío de información que dificulta considerar y comprender el fenómeno de comorbilidad en la práctica clínica, lo cual impacta directamente al momento de intentar responder a las necesidades del paciente y su familia. Si bien se ha realizado un importante esfuerzo por detectar oportunamente la comorbilidad psiquiátrica con trastornos por consumo de sustancias, todavía son pocos los estudios reportados en México.

Es por esto que el presente estudio se realizó en los CRAMAA, maniobra que permitirá tener un panorama más específico de la población que acude a estos centros y de sus necesidades de atención. De tal forma, se pretende proporcionar información de primera mano que coadyuve en la creación de políticas públicas, que se traduzcan en tratamientos más especializados para la población estudiada, para reducir costos e incrementar la efectividad de los mismos.

\section{METODOLOGÍA}

\section{Diseño del estudio}

Se realizó un estudio transversal en dos Centros Residenciales de Ayuda-Mutua para la Atención de las Adicciones (CRAMAA) en el sur del Distrito Federal, con participantes que fueron diagnosticados a su ingreso con trastornos por consumo de sustancias. El trabajo de campo se realizó en una ventana de tiempo de 24 meses, misma que se dividió en dos etapas. La primera (Etapa 1) consistió en una evaluación inicial, realizada por psiquiatras, para identificar y eliminar del estudio, de acuerdo con los criterios de exclusión, a aquellos participantes en los que se detectara la presencia de sintomatología psicótica, maniaca y/o de deterioro cognitivo que limitara su capacidad de responder a la entrevista estructurada de la etapa siguiente.

La segunda etapa (Etapa 2) consistió en la aplicación de una entrevista estructurada, asistida por psicólogos capacitados en la aplicación del instrumento en modalidad computarizada, donde se evaluó la presencia de los trastornos psiquiátricos a lo largo de la vida, según los criterios diagnósticos del Manual Diagnóstico y Estadístico de los Trastornos Mentales- $I V$, texto revisado (DSM-IV-TR). ${ }^{27}$

\section{Participantes}

Se realizó un muestreo no probabilístico por conveniencia de 535 participantes del sexo masculino, que se encontraban 


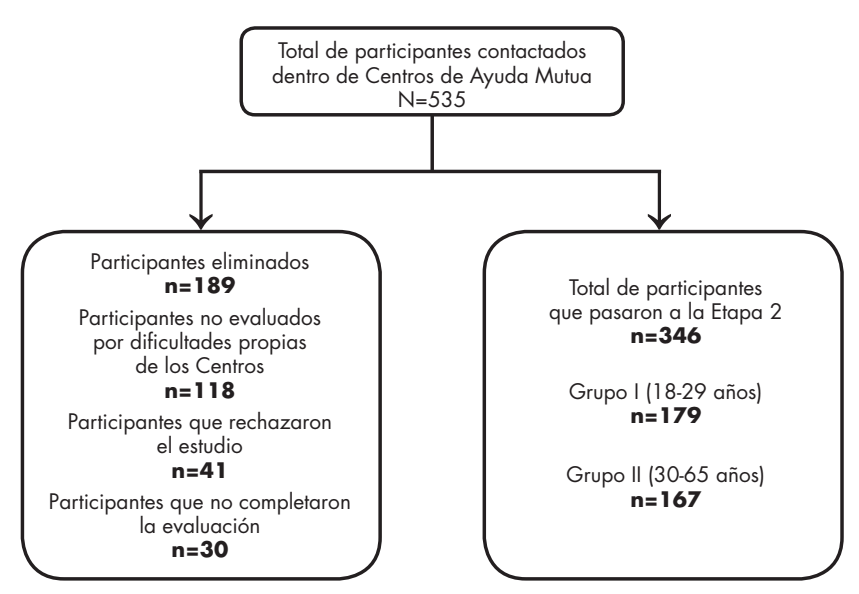

Figura 1. Diagrama del estudio.

en internamiento en los dos CRAMAA seleccionados. Éstos debían cumplir con los siguientes criterios de inclusión: a) tener entre 18 y 65 años de edad, b) aceptar participar voluntariamente en el estudio, c) presentar algún diagnóstico por consumo de sustancias, d) saber leer y escribir y e) contar con un familiar responsable que diera su consentimiento informado para la inclusión del participante en el protocolo de investigación.

\section{Instrumentos}

En la etapa 1 se aplicó una entrevista clínica y una batería clinimétrica de apoyo para el psiquiatra, la cual fue integrada por la escala de Manía de Young, ${ }^{28-30}$ la sección de psicosis de la Entrevista Diagnóstica Internacional Compuesta versión en papel 3.0 (CIDI) y el Mini-Examen Cognoscitivo MEC-35,31,32 con el objetivo de identificar sintomatología maniaca, psicótica y/o de deterioro cognitivo que incapacitara al participante a continuar con el estudio.

En la etapa 2 se aplicó la versión computarizada del $\mathrm{CIDI}^{33,34}$ la cual es una entrevista completamente estructurada que proporciona diagnósticos según los criterios del DSM-IV-TR para los trastornos por consumo de sustancias y otros 17 trastornos psiquiátricos. Este instrumento ha sido adaptado y validado a nivel internacional, además de ser empleado en la encuesta de Comorbilidad Psiquiátrica de México. $^{35}$

\section{Procedimiento}

Se realizó la selección de sedes con base en los criterios de certificación y funcionamiento que señala la Secretaría de Salud en la Norma Oficial Mexicana NOM028 SSA2-2009 para la Prevención, Tratamiento y Control de las Adicciones para Centros de Tratamiento Residenciales de Ayuda-Mutua.

Previo al trabajo de campo se capacitó a entrevistadores legos (pasantes de psicología) en la aplicación de la versión computarizada de la Entrevista Diagnóstica Internacional Compuesta versión 3.0 (CIDI), así como el entrenamiento en aplicación de escalas clinimétricas a los psiquiatras.

Durante el trabajo de campo se proporcionó una explicación verbal y escrita del estudio a cada participante y a su familiar responsable, y se obtuvo el consentimiento de ambos para proceder con la entrevista. Los participantes que cumplieron los criterios de inclusión pasaron a la etapa 2.

Al finalizar las entrevistas, se realizó una plática psicoeducativa, que tuvo por objetivo explicar qué es la comorbilidad de los trastornos por consumo de sustancias con otros trastornos psiquiátricos y las implicaciones en el tratamiento, además de proporcionar las alternativas de referencia y derivación a servicios de atención especializada.

\section{RESULTADOS}

La muestra total se integró por 535 participantes de sexo masculino, de los cuales 189 fueron eliminados del estudio y 346 fueron evaluados con el CIDI. En función de la alta prevalencia e incidencia en el consumo de sustancias entre la población joven, se dividió la muestra en dos grupos a fin de realizar un análisis de la prevalencia de comorbilidad tomando en consideración la variable de edad. De esta forma, 179 participantes pertenecen al Grupo I (18 a 29 años) y 167 pertenecen al Grupo II (30 a 65 años) (figura 1).

Las características demográficas de la muestra se presentan en la cuadro 2. La escolaridad estaba distribuida de manera similar entre aquellos con 7-9 años y más de 12 años de estudio; en su mayoría, los participantes habían estudiado entre 10 y 12 años y sólo una pequeña proporción tenía entre cero y seis años de escolaridad. En relación con el estado civil, dos terceras partes reportaron que eran separados, divorciados, viudos o, incluso, que nunca habían estado casados; de estos últimos se observó que más de la mitad no tenían una relación de pareja al momento de ser entrevistados. Por otra parte, se encontró que la situación laboral más frecuente fue el autoempleo y subempleo, distribuidas de manera similar (cuadro 2).

La sustancia principal de consumo fue alcohol (43\%), seguido de cocaína (25\%) y marihuana, aunque esta última en menor porcentaje (17\%). Toda la muestra reportó tener al menos uno de los trastornos por consumo de sustancias, siendo el trastorno por dependencia al alcohol el más frecuente "alguna vez en la vida" (63.01\%), seguido por el trastorno por dependencia a drogas $(57.51 \%)$.

Por otra parte, al comparar los grupos de edad se encontró que en el Grupo II, el de mayor edad (30-65 años), se observa una prevalencia mayor en el trastorno por dependencia al alcohol (70.66\%) $\left(\mathrm{X}^{2}=8.111, g l=1, \mathrm{p} \leq 0.05\right)$; mientras que para el Grupo I, el de menor edad (18-29 años), el trastorno por dependencia a drogas (70.95\%) $\left(X^{2}=27.396\right.$, $g l=1, \mathrm{p} \leq 0.05)$ fue el más prevalente. Asímismo, los análisis 
Cuadro 2. Principales variables demográficas

\begin{tabular}{|c|c|c|c|}
\hline & $\mathrm{n}$ & $\mathrm{F}$ & $\%$ \\
\hline Sexo: Hombre & 346 & 346 & 100.0 \\
\hline $\begin{array}{l}\text { Edad } \\
\text { - } 18 \text { a } 19 \\
\text { - } 20 \text { a } 29 \\
\text { - } 30 \text { a } 39 \\
\text { - } 40 \text { o más }\end{array}$ & 346 & $\begin{array}{r}52 \\
127 \\
87 \\
80\end{array}$ & $\begin{array}{l}15.0 \\
36.7 \\
25.1 \\
23.1\end{array}$ \\
\hline $\begin{array}{l}\text { Escolaridad } \\
\text { - } 0 \text { a } 6 \text { años } \\
\text { - } 7 \text { a } 9 \text { años } \\
\text { - } 10 \text { a } 12 \text { años } \\
\text { - Más de } 12 \text { años }\end{array}$ & 346 & $\begin{array}{r}23 \\
96 \\
127 \\
100\end{array}$ & $\begin{array}{r}6.9 \\
28.0 \\
36.9 \\
28.9\end{array}$ \\
\hline $\begin{array}{l}\text { Estado civil } \\
\text { - Casado / Unión libre } \\
\text { - Separado / Divorciado / Viudo } \\
\text { - Nunca casado }\end{array}$ & 346 & $\begin{array}{r}83 \\
69 \\
194\end{array}$ & $\begin{array}{l}24.0 \\
19.9 \\
56.1\end{array}$ \\
\hline $\begin{array}{l}\text { Actual relación de pareja no } \\
\text { (estado civil soltero y con rel } \\
\text { pareja actual) } \\
\text { - Sí } \\
\text { - No }\end{array}$ & nial: & $\begin{array}{r}61 \\
202\end{array}$ & $\begin{array}{l}17.6 \\
58.4\end{array}$ \\
\hline $\begin{array}{l}\text { Situación laboral } \\
\text { - Empleado } \\
\text { - Autoempleado } \\
\text { - Subempleado } \\
\text { - Desempleado }\end{array}$ & 346 & $\begin{array}{r}89 \\
118 \\
111 \\
28\end{array}$ & $\begin{array}{r}25.8 \\
34.0 \\
32.0 \\
8.2\end{array}$ \\
\hline
\end{tabular}

mostraron diferencias estadísticamente significativas en las proporciones de ambos grupos (cuadro 3).

Se evaluaron un total de 17 trastornos psiquiátricos del Eje I, además de los trastornos por consumo de sustancias, y se agruparon de acuerdo con los grupos diagnósticos del DSMIV-TR. De este modo quedaron conformados en: "Trastornos afectivos, Trastornos de ansiedad, Trastornos del control de impulsos (Juego patológico), Trastornos de la conducta alimentaria, Trastornos por déficit de atención y comportamiento perturbador y Trastornos de ansiedad de separación".

Con respecto a la comorbilidad de trastornos por consumo de sustancias con otros trastornos psiquiátricos, se observó que tres cuartas partes de la población evaluada
(75.72\%) cumplía con criterios diagnósticos para cualquier trastorno mental, alguna vez en la vida. El grupo de trastornos comórbidos más frecuente fue el déficit de atención y comportamiento perturbador $(56.94 \%)$, seguido por los trastornos de ansiedad (30.35\%), de ansiedad de separación $(24.28 \%)$, afectivos $(23.99 \%)$, de control de los impulsos $(12.14 \%)$ y de la conducta alimentaria (6.07\%) (cuadro 4). El trastorno disocial y el trastorno oposicionista desafiante se destacan por ser los trastornos individuales más frecuentes.

Comparando ambos grupos de edad, se encontró una diferencia estadísticamente significativa en la proporción de participantes que presentaron trastornos de control de los impulsos $\left(X^{2}=4.269, g l=1, p \leq 0.05\right)$, trastornos por déficit de atención y comportamiento perturbador $\left(X^{2}=23.023\right.$, $g l=1, p \leq 0.05)$ y cualquier trastorno psiquiátrico del Eje I $\left(X^{2}=13.159, g l=1, p \leq 0.05\right)$.

Se observó que el Grupo I (18-29 años) presentó un porcentaje mayor en la presencia de comorbilidad para trastornos de control de los impulsos (15.64\%), trastornos por déficit de atención y comportamiento perturbador $(69.27 \%)$ y para cualquier trastorno psiquiátrico $(83.80 \%)$ en comparación con el Grupo II (30-65 años) (67.07\%) (cuadro 4).

$\mathrm{Al}$ analizar la comorbilidad por grupos de trastornos por consumo de sustancias, se evidenció que los relacionados por consumo de alcohol presentan relaciones con diferencias estadísticamente significativas entre "abuso de alcohol y cualquier trastorno psiquiátrico $\left(X^{2}=6.335, g l=1\right.$, $\mathrm{p} \leq 0.05)$; abuso de alcohol y trastornos afectivos $\left(X^{2}=4.408\right.$, $g l=1, \mathrm{p} \leq 0.05)$ y dependencia al alcohol y trastornos de ansiedad $\left(X^{2}=5.685, g l=1, p \leq 0.05\right) "$ (cuadro 5).

En relación con los participantes que presentaron abuso de drogas, no se encontró una diferencia estadísticamente significativa para ningún grupo de trastornos, en comparación con los participantes que presentaron dependencia a drogas, donde se encontró diferencia para los grupos de "trastornos afectivos $\left(X^{2}=6.832, g l=1, p \leq 0.05\right)$, de ansiedad $\left(X^{2}=7.542, g l=1, p \leq 0.05\right)$, de control de los impulsos $\left(X^{2}=10.747, g l=1, p \leq 0.05\right)$, déficit de atención y comportamiento perturbador $\left(X^{2}=31.853, g l=1, p \leq 0.05\right)$ y cualquier trastorno psiquiátrico $\left(X^{2}=26.545, g l=1, p \leq 0.05\right)$ " (cuadro 5).

Cuadro 3. Prevalencia de los trastornos por consumo de sustancias (Alguna vez en la vida) por grupos de edad

\begin{tabular}{|c|c|c|c|c|c|c|}
\hline \multirow[b]{2}{*}{ Trastornos } & \multicolumn{2}{|c|}{$\begin{array}{c}\text { Grupo I } \\
\text { (18-29 años) } \\
(n=179)\end{array}$} & \multicolumn{2}{|c|}{$\begin{array}{c}\text { Grupo II } \\
\text { (30-65 años) } \\
(n=167)\end{array}$} & \multicolumn{2}{|c|}{$\begin{array}{l}\text { Muestra total } \\
\quad(n=346)\end{array}$} \\
\hline & $\mathrm{F}$ & $\%$ & $\mathrm{~F}$ & $\%$ & $\mathrm{~F}$ & $\%$ \\
\hline Por consumo de sustancias & 179 & 100.00 & 167 & 100.00 & 346 & 100.00 \\
\hline Por abuso de alcohol & 46 & 25.70 & 38 & 22.75 & 84 & 24.28 \\
\hline Por dependencia a alcohol* & 100 & 55.87 & 118 & 70.66 & 218 & 63.01 \\
\hline Por abuso de drogas & 30 & 16.76 & 22 & 13.17 & 52 & 15.03 \\
\hline Por dependencia a drogas* & 127 & 70.95 & 72 & 43.11 & 199 & 57.51 \\
\hline
\end{tabular}

*Indica diferencias significativas ( $\mathrm{p} \leq 0.05) ; \mathrm{F}=$ Frecuencia. 
Cuadro 4. Prevalencia de los trastornos psiquiátricos del Eje I (Alguna vez en la vida) por grupos de edad

\begin{tabular}{|c|c|c|c|c|c|c|}
\hline \multirow[b]{2}{*}{ Trastornos } & \multicolumn{2}{|c|}{$\begin{array}{c}\text { Grupo I } \\
\text { (18-29 años) } \\
n=179 \\
\end{array}$} & \multicolumn{2}{|c|}{$\begin{array}{c}\text { Grupo II } \\
\text { (30-65 años) } \\
n=167 \\
\end{array}$} & \multicolumn{2}{|c|}{$\begin{array}{l}\text { Muestra total } \\
n=346\end{array}$} \\
\hline & $\mathrm{F}$ & $\%$ & $\mathrm{~F}$ & $\%$ & $\mathrm{~F}$ & $\%$ \\
\hline Trastornos afectivos & 43 & 24.02 & 40 & 23.95 & 83 & 23.99 \\
\hline - Trastorno depresivo mayor & 33 & 18.44 & 29 & 17.37 & 62 & 17.92 \\
\hline - Trastorno distímico & 7 & 3.91 & 7 & 4.19 & 14 & 4.05 \\
\hline - Trastornos afectivos bipolares I y II & 10 & 5.59 & 7 & 4.19 & 17 & 4.91 \\
\hline Trastornos de ansiedad & 59 & 32.96 & 46 & 27.54 & 105 & 30.35 \\
\hline - Trastorno de angustia & 11 & 6.15 & 1 & 0.60 & 12 & 3.47 \\
\hline - Agorafobia & 3 & 1.68 & 3 & 1.80 & 6 & 1.73 \\
\hline - Fobia social & 32 & 17.88 & 20 & 11.98 & 52 & 15.03 \\
\hline - Trastorno obsesivo compulsivo & 4 & 2.23 & 5 & 2.99 & 9 & 2.60 \\
\hline - Trastorno por estrés postraumático & 23 & 12.85 & 22 & 13.17 & 45 & 13.01 \\
\hline - Trastorno de ansiedad generalizada & 12 & 6.70 & 5 & 2.99 & 17 & 4.91 \\
\hline Trastornos de control de impulsos* & 28 & 15.64 & 14 & 8.38 & 42 & 12.14 \\
\hline - Juego patológico & 28 & 15.64 & 14 & 8.38 & 42 & 12.14 \\
\hline Trastornos de la conducta alimentaria & 15 & 08.38 & 6 & 3.59 & 21 & 6.07 \\
\hline - Anorexia & 3 & 1.68 & 0 & 0 & 3 & 0.87 \\
\hline - Bulimia & 13 & 7.26 & 6 & 3.59 & 19 & 5.49 \\
\hline $\begin{array}{l}\text { Trastornos por déficit de atención } \\
\text { y comportamiento perturbador* }\end{array}$ & 124 & 69.27 & 73 & 43.71 & 197 & 56.94 \\
\hline $\begin{array}{l}\text { - Trastorno por déficit de atención } \\
\text { e hiperactividad }\end{array}$ & 48 & 26.82 & 22 & 13.17 & 70 & 20.23 \\
\hline - Trastorno disocial & 100 & 55.87 & 53 & 31.74 & 153 & 44.22 \\
\hline - Trastorno oposicionista desafiante & 76 & 42.46 & 34 & 20.36 & 110 & 31.79 \\
\hline Trastornos de ansiedad de separación & 44 & 24.58 & 40 & 23.95 & 84 & 24.28 \\
\hline - Ansiedad de separación adultos & 39 & 21.79 & 35 & 20.96 & 74 & 21.39 \\
\hline - Ansiedad de separación infancia & 15 & 8.38 & 11 & 6.59 & 26 & 7.51 \\
\hline Cualquier trastorno psiquiátrico* & 150 & 83.80 & 112 & 67.07 & 262 & 75.72 \\
\hline
\end{tabular}

*Indica diferencias significativas $(p \leq 0.05) ; F=$ Frecuencia.

Finalmente, las frecuencias de comorbilidad psiquiátrica presentaron mayor prevalencia en los trastornos por dependencias (dependencia a drogas [85.93\%], dependencia al alcohol $[78.90 \%])$; frente a los trastornos por abuso (abuso de drogas [75\%] y abuso de alcohol [65.48\%]), lo cual confirma la hipótesis asociativa que señala que a mayor gravedad de la adicción, mayor comorbilidad psiquiátrica (cuadro 5).

Por otra parte, se encontró que más de $70 \%$ de la muestra con comorbilidad psiquiátrica presentó más de dos trastornos psiquiátricos comórbidos del Eje I, lo cual indica que esta población tiende a presentar síndromes psiquiátricos complejos. Aunado a ésto, al comparar por grupos de edad, se evidencia que el grupo de menor edad (Grupo I) presenta un mayor número de trastornos psiquiátricos comórbidos, en comparación con el grupo de mayor edad (Grupo II). De esta forma se alcanza una diferencia estadísticamente significativa $(\mathrm{t}=4.385, g l=344, p \leq 0.05)$, donde la media para el Grupo I (18-29 años) fue de $\bar{X}=2.55$, mientras que para el Grupo II ( 30 a 65 años) la media fue de $\bar{X}=1.64$ (cuadro 6).

Asimismo, se realizó una comparación en cuanto al orden de aparición de los trastornos psiquiátricos (previo, simultáneo o posterior) en relación con los trastornos por consumo de sustancias, con lo que se encontraron diferencias estadísticamente significativas para cualquier trastorno psiquiátrico $\left(\chi^{2}=302.573, g l=2, p \leq 0.05\right)$. Por esto, y de acuerdo con el resultado, se logró identificar que más de dos terceras partes $(83.59 \%)$ de la muestra evaluada iniciaron con síntomas psiquiátricos previos a la aparición de síntomas adictivos. Es decir, este hallazgo demuestra y confirma que en su gran mayoría los trastornos psiquiátricos se presentan de forma más temprana en relación con los trastornos adictivos (cuadro 7).

Posteriormente, se buscaron asociaciones por cada grupo de trastornos y se encontró que todos los grupos de trastornos psiquiátricos mostraron diferencias estadísticamente significativas. Sin embargo, el que fue predominantemente mayor, en función de la prevalencia, fue el "grupo de trastorno por déficit de atención y comportamiento perturbador $\left(\chi^{2}=304.335, g l=2, p \leq 0.05\right) "$ (cuadro 7).

\section{DISCUSIÓN}

Los resultados obtenidos en el presente estudio son consistentes con los resultados reportados en la literatura internacional $^{8-17}$ para centros de atención a las adicciones, a pesar de que éste se realizó en un modelo de atención de AyudaMutua, que no es común en otros países. Este hallazgo hace 
Cuadro 5. Prevalencia de trastornos psiquiátricos comórbidos del Eje I con trastornos por consumo de sustancias

\begin{tabular}{|c|c|c|c|c|c|c|c|c|}
\hline & \multicolumn{8}{|c|}{ Trastorno por } \\
\hline & \multicolumn{2}{|c|}{$\begin{array}{c}\text { Abuso de alcohol } \\
\mathrm{n}=84\end{array}$} & \multicolumn{2}{|c|}{$\begin{array}{l}\text { Dependencia de alcohol } \\
n=218\end{array}$} & \multicolumn{2}{|c|}{$\begin{array}{c}\text { Abuso a drogas } \\
\mathrm{n}=52\end{array}$} & \multicolumn{2}{|c|}{$\begin{array}{c}\begin{array}{c}\text { Dependencia a drogas } \\
\mathrm{n}=199\end{array} \\
\end{array}$} \\
\hline & $\mathrm{n}$ & $\%$ & $\mathrm{n}$ & $\%$ & $\mathrm{n}$ & $\%$ & $\mathrm{n}$ & $\%$ \\
\hline Trastornos afectivos & 13 & 15.48 * & 55 & 25.23 & 8 & 15.38 & 58 & $29.15^{*}$ \\
\hline - Trastorno depresivo mayor & 8 & 9.52 & 39 & 17.88 & 7 & 13.46 & 43 & 21.61 \\
\hline - Trastorno distímico & 3 & 3.57 & 7 & 3.21 & 2 & 3.85 & 11 & 5.53 \\
\hline - Trastornos afectivos bipolares I y II & 5 & 5.95 & 11 & 5.05 & 1 & 1.92 & 12 & 6.03 \\
\hline Trastornos de ansiedad & 21 & 25.00 & 76 & $34.86^{*}$ & 15 & 28.85 & 72 & $36.18 *$ \\
\hline - Trastorno de angustia & 4 & 4.76 & 6 & 2.75 & 1 & 1.92 & 10 & 5.03 \\
\hline - Agorafobia & 0 & 2.38 & 11 & 5.05 & 1 & 1.92 & 9 & 4.52 \\
\hline - Fobia social & 9 & 10.71 & 38 & 17.43 & 6 & 11.54 & 39 & 19.60 \\
\hline - Trastorno obsesivo compulsivo & 3 & 3.57 & 5 & 2.29 & 3 & 5.77 & 2 & 1.01 \\
\hline - Trastorno por estrés postraumático & 4 & 4.76 & 39 & 17.89 & 4 & 7.69 & 32 & 16.10 \\
\hline - Trastorno de ansiedad generalizada & 6 & 7.14 & 10 & 4.59 & 4 & 7.69 & 11 & 5.53 \\
\hline Trastornos de control de impulsos & 9 & 10.71 & 26 & 11.93 & 5 & 9.62 & 34 & $17.09 *$ \\
\hline - Juego patológico & 9 & 10.71 & 26 & 11.96 & 5 & 9.62 & 34 & 17.09 \\
\hline Trastornos de la conducta alimentaria & 2 & 2.38 & 16 & 7.34 & 1 & 1.92 & 14 & 7.03 \\
\hline - Anorexia & 1 & 1.19 & 2 & 0.92 & 0 & 0 & 1 & 0.50 \\
\hline - Bulimia & 1 & 1.19 & 15 & 6.88 & 1 & 1.92 & 13 & 6.53 \\
\hline $\begin{array}{l}\text { Trastornos por déficit de atención } \\
\text { y comportamiento perturbador }\end{array}$ & 43 & 51.19 & 129 & 59.17 & 29 & 55.77 & 139 & $69.85^{*}$ \\
\hline $\begin{array}{l}\text { - Trastorno por déficit de atención } \\
\text { e hiperactividad }\end{array}$ & 16 & 19.04 & 44 & 20.18 & 7 & 13.46 & 52 & 26.13 \\
\hline - Trastorno disocial & 33 & 39.28 & 103 & 47.25 & 24 & 46.15 & 110 & 55.28 \\
\hline - Trastorno oposicionista desafiante & 30 & 35.71 & 70 & 32.11 & 11 & 21.15 & 87 & 43.72 \\
\hline Trastornos de ansiedad de separación & 18 & 21.43 & 49 & 22.47 & 13 & 25.00 & 49 & 24.62 \\
\hline - Ansiedad de separación adultos & 14 & 16.67 & 49 & 22.47 & 13 & 25.00 & 49 & 24.62 \\
\hline - Ansiedad de separación infancia & 7 & 8.33 & 17 & 7.80 & 2 & 3.85 & 18 & 9.04 \\
\hline Cualquier trastorno psiquiátrico & 55 & $65.48^{*}$ & 172 & 78.90 & 39 & 75.00 & 171 & $85.93 *$ \\
\hline
\end{tabular}

*Indica diferencias significativas $(p \leq 0.05)$.

hincapié en las importantes necesidades de atención de dicha población de difícil acceso (CRAMAA), ya que, como se ha mencionado, más de $75 \%$ de los participantes evaluados presentaron comorbilidad psiquiátrica y en su mayoría el tratamiento a esta comorbilidad es limitado o nulo, ,3,19 así como la información sobre la misma.

Por otro lado, comparando los resultados obtenidos en el estudio con los reportado en población abierta por Caraveo-Anduaga y Colmenares-Bermúdez (2002), se puede observar que los trastornos de ansiedad y afectivos resultan

Cuadro 6. Número de trastornos psiquiátricos comórbidos del Eje I con cualquier trastorno por consumo de sustancias por grupo de edad

\begin{tabular}{|c|c|c|c|c|c|c|}
\hline & \multicolumn{2}{|c|}{$\begin{array}{c}\text { Grupo I } \\
\text { (18-29 años) } \\
n=179 \\
\end{array}$} & \multicolumn{2}{|c|}{$\begin{array}{c}\text { Grupo II } \\
\text { (30-65 años) } \\
n=167 \\
\end{array}$} & \multicolumn{2}{|c|}{$\begin{array}{c}\text { Muestra tota } \\
\begin{array}{c}\text { (18-65 años) } \\
n=346\end{array}\end{array}$} \\
\hline & $\mathrm{F}$ & $\%$ & $\mathrm{~F}$ & $\%$ & $\mathrm{~F}$ & $\%$ \\
\hline Sin comorbilidad & 29 & 16.2 & 55 & 32.9 & 84 & 24.3 \\
\hline 1 & 31 & 17.3 & 44 & 26.4 & 75 & 21.7 \\
\hline 2 & 35 & 19.6 & 33 & 20.0 & 68 & 19.7 \\
\hline 3 & 34 & 19.0 & 11 & 7.0 & 45 & 13.0 \\
\hline 4 & 26 & 14.5 & 9 & 5.4 & 35 & 10.1 \\
\hline 5 o más & 24 & 13.4 & 15 & 9.0 & 39 & 11.3 \\
\hline
\end{tabular}

ser los más prevalentes en comorbilidad con los trastornos por consumo de sustancias. ${ }^{22}$ No obstante, en el presente estudio es relevante mencionar que el grupo de trastornos del Eje I con mayor prevalencia fue el trastorno por déficit de atención y comportamiento perturbador (56.94\%), lo cual indica una tendencia importante a ser replanteada en futuros estudios, con el objetivo de desarrollar estrategias de tratamiento sensibles a las necesidades de esta población.

Es importante señalar que el Grupo I (18-29 años) presentó mayor comorbilidad psiquiátrica (83.80\%) y dependencia a drogas (70.95\%), en comparación con el Grupo II (30-65 años), en el cual la comorbilidad fue menor $(67.07 \%)$ y la sustancia de mayor impacto fue el alcohol (70.66\%). Esto podría representar un hallazgo relevante, ya que los jóvenes presentan mayor comorbilidad, lo cual podría verse influido por la sustancia de preferencia y la cantidad y frecuencia de consumo.

Cabe mencionar que este estudio no se diseñó para establecer relaciones de causalidad. Sin embargo, fue posible conocer que la frecuencia en relación con el inicio de síntomas psiquiátricos como antecedentes a los síntomas adictivos es mayor (83.59\%), lo cual corresponde con los resultados de estudios en México realizados en población abierta. ${ }^{19}$ Es por lo anterior que los trastornos de inicio en la infancia 
Cuadro 7. Edad de inicio de síntomas psiquiátricos del Eje I en relación a la edad de inicio de síntomas adictivos

\begin{tabular}{|c|c|c|c|c|c|c|c|}
\hline \multirow[b]{3}{*}{ Trastornos } & \multirow[b]{3}{*}{ N } & \multicolumn{6}{|c|}{ Inicio de síntomas psiquiátricos } \\
\hline & & \multicolumn{2}{|c|}{$\begin{array}{c}\text { Previo } \\
\text { a los síntomas } \\
\text { adictivos }\end{array}$} & \multicolumn{2}{|c|}{$\begin{array}{l}\text { Simultáneo } \\
\text { a los síntomas } \\
\text { adictivos }\end{array}$} & \multicolumn{2}{|c|}{$\begin{array}{c}\text { Posterior } \\
\text { a los síntomas } \\
\text { adictivos }\end{array}$} \\
\hline & & $\mathrm{F}$ & $\%$ & $\mathrm{~F}$ & $\%$ & $\mathrm{~F}$ & $\%$ \\
\hline Afectivos* & 83 & 36 & 43.37 & 9 & 10.84 & 38 & 45.78 \\
\hline Ansiedad* & 105 & 65 & 61.90 & 12 & 11.43 & 28 & 26.67 \\
\hline Control de impulsos* & 42 & 9 & 21.43 & 6 & 14.29 & 27 & 64.29 \\
\hline Conducta alimentaria* & 21 & 11 & 52.38 & 2 & 9.52 & 8 & 38.10 \\
\hline Déficit de atención y comportamiento perturbador* & 197 & 181 & 91.88 & 4 & 2.03 & 12 & 6.09 \\
\hline Ansiedad por separación* & 84 & 47 & 55.95 & 2 & 2.38 & 35 & 41.67 \\
\hline Cualquier trastorno psiquiátrico* & 262 & 219 & 83.59 & 7 & 2.67 & 36 & 13.74 \\
\hline
\end{tabular}

*Indica diferencias Significativas ( $\mathrm{p} \leq 0.05) ; \mathrm{F}=$ Frecuencia.

se deben detectar y atender oportunamente, ya que, como se encontró en este estudio, representan una frecuente entidad patológica que antecede al consumo de sustancias. De este modo, la atención oportuna puede ser probablemente una estrategia efectiva de prevención contra el desarrollo de trastornos adictivos.

Finalmente, se encontró que más de $70 \%$ de esta población presenta más de dos trastornos psiquiátricos del Eje I, a lo largo de la vida, cifra por demás significativa, aun sin haber considerado dentro del estudio la evaluación del Eje II (trastornos de la personalidad), y personas con sintomatología maniaca, psicótica y/o de deterioro cognitivo. Lo anterior nos permite conocer que esta población presenta una evolución de la psicopatología, lo que implica la presencia de síndromes psiquiátricos complejos de difícil atención. Dentro de las limitaciones del estudio es importante señalar que si bien la muestra no es representativa de la población de los CRAMAA a nivel nacional, se puede considerar como una primera aproximación al entendimiento de la comorbilidad de trastornos psiquiátricos en personas que asisten a estos centros en busca de atención a causa de su consumo de sustancias.

Otra de las limitaciones es que fue realizado únicamente con participantes de sexo masculino, lo cual limita el conocimiento de este fenómeno en población de sexo femenino. Sin embargo, puede constituir una línea de investigación para futuros estudios, ya que la literatura internacional reporta una importante prevalencia de trastornos psiquiátricos, situaciones de trauma y conducta suicida, en las mujeres que presentan trastornos por consumo de sustancias. ${ }^{11}$ Los hallazgos de este estudio poseen un valor significativo en al menos tres directrices: Primero, la creación de nuevas hipótesis de investigación acerca de la etiopatología de los trastornos por consumo de sustancias.

En segunda instancia, éste representa un indicador de relevancia para la creación de modelos de tratamiento que contemplen la comorbilidad psiquiátrica. Cabe señalar que la carencia de programas de tratamiento que integren la atención psiquiátrica y para las adicciones, como es el caso de los CRAMAA, que no cuentan con servicios profesionales, incrementa el deterioro biopsicosocial, además de presentar una inadecuada respuesta al tratamiento.

Es importante hacer hincapié en la necesidad de proporcionar una atención integral y multidisciplinaria en los diferentes escenarios de tratamiento, ya que el presente estudio hace evidente la urgencia de considerar la existencia de padecimientos psiquiátricos comórbidos al consumo de sustancias, a fin de incrementar la eficacia y reducir los costos. Ello incrementaría la probabilidad de éxito del tratamiento y reduciría el índice de recaídas, situación que se espera se traduzca en el mejoramiento de la calidad de vida del paciente y su familia.

En tercer lugar, los resultados del presente estudio podrían constituir un indicador que coadyuve a la creación de políticas públicas en el campo de la prevención y/o tratamiento de las adicciones. Si bien es cierto que los CRAMAA han representado una alternativa para muchas personas, también es relevante señalar que muchas de estas agrupaciones violan la Ley General de Salud al operar sin equipo, personal e infraestructura adecuados según los lineamientos marcados por la Norma Oficial Mexicana NOM-028SSA2-2009 para la Prevención, Tratamiento y Control de las Adicciones.

Asímismo, este estudio viene a ser el primero dedicado a la evaluación de la prevalencia de comorbilidad de los trastornos mentales en la población cautiva de Centros Residenciales de Ayuda-Mutua para la Atención de las Adicciones y uno de los pocos realizados en centros de atención para las adicciones en México.

\section{AGRADECIMIENTOS}

Se extiende un especial agradecimiento a los equipos de evaluación conformados por psiquiatras y psicólogos, que con tanto entusiasmo participaron para el levantamiento de la información presentada en este estudio, así como a los científicos y expertos que aportaron su conocimiento para el análisis y presentación de los resultados. 


\section{REFERENCIAS}

1. Medina-Mora ME, Natera G, Borges G, Cravioto P et al. Del siglo XX al tercer milenio. Las adicciones y la salud pública: drogas, alcohol y sociedad. Salud Mental 2001;24:3-19.

2. Daley D, Moss H. Dual disorder: Counseling clients with chemical dependency and mental illness. Tercera edición.) Minnesota: Hazelden; 2002.

3. Substance Abuse and Mental Health Services Administration (SAMHSA). Report to congress on the prevention and treatment of cooccurring substance abuse disorders and mental disorders. Inventory number: BKD467; 2002.

4. Substance Abuse and Mental Health Services Administration (SAMHSA) and Center for Substance Abuse Center (CSAT). Substance abuse treatment for persons with co-occurring disorders. Treatment improvement protocol Vol. 42. [DHHS Publication No. (SMA) 05-3992]; 2005.

5. Regier DA, Farmer ME, Rae DS, Locke BZ et al. Comorbidity of mental disorders with alcohol and other drug abuse. Results from the epidemiologic catchment area (ECA) study. JAMA 1990;264(19):2511-2518.

6. World Health Organizations. The world health report 2001. Metal Health: New Understanding, New Hope. Ginebra: 2001.

7. Levounis P, Galanter M, Dermatis H, Hamowy A et al. Correlates of HIV transmission risk factors and considerations for interventions in homeless, chemically addicted and mentally ill patients. J Addictive Diseases 2002;61-72.

8. Lukasiewicz M, Blecha L, Falissard BXN, Benyamina A et al. Dual diagnosis: Prevalence, risk factors, and relationship with suicide risk in a nationwide sample of French prisioners. Alcoholism: Clinical Experimental Research 2009;160-168.

9. Young DS. Co-occurring disorders among jail inmates: Bridging the treatment gap. J Social Work Practice Addictions 2000;63-85.

10. Slesnick N, Prestopnik J. Dual and multiple diagnosis among substance using runaway youth. American J Drug Alcohol Abuse 2005;179201.

11. Newmann JP, Sallman J. Women, trauma histories, and co-occurring disorders: Assessing the scope of the problem. Social Service Review 2004;466-499.

12. Rush B, Koegl CJ. Prevalence and Profile of People with Co-occurring Mental and Substance Use Disorders within a Comprehensive Mental Health System. Canadian Journal of Psychiatry, 2008;810-821.

13. Swadi H, Bobier C. Substance use disorder comorbidity among inpatient youths with psychiatric disorder. Australian New Zealand J Psychiatry, 2003;294-298.

14. Nocon A, Bergé D, Astals M, Martín-Santos R et al. Dual diagnosis in an inpatient drug-abuse detoxification unit. European Addiction Research 2007;192-200.

15. Watkins KE, Hunter SB, Wenzel SL, Tu W et al. Prevalence and characteristics of clients with co-occurring disorders in outpatient substance abuse treatment. American J Drug Alcohol Abuse 2004;749-764.

16. Fabricius V, Langa M, Wilson K. An exploratory investigation of cooccurring substance related and psychiatric disorders. J Substance Use 2008;99-114.

17. Hasin D, Nunes E, Meylan J. Comorbidity of alcohol, drug and psychiatric disorders: Epidemiology. En: Kranzler H, Tinsley J. Dual diagnosis and psychiatric treatment: Substance abuse and comorbid disorders. USA: Segunda edición; Marcel Bekker; 2004; pp. 1-34.
18. Drake RE, Wallach MA. Dual diagnosis: 15 Years of Progress. Psychiatric Services 51(9):1126-1129.

19. Sacks S, Sacks J, De Leon G, Bernhardt AI et al. Modified therapeutic community for mentally ill Chemicals "abusers": Background; influences; program description; preliminary findings. Substance Use Misuse 1997;32(9):1217-1225.

20. Brunette MF, Mueser KT, Drake RE. A review of research on residential programs for people with severe mental illness and co-occurring substance use disorders. Drug Alcohol Review 2004;23:471-481.

21. Drake RE, Mueser KT, Brunette MF, McHugo GJ. A Review of treatments for people with severe mental illnesses and co-occurring substance use disorders. Psychiatric Rehabilitation J, 2004;27(4):360-374.

22. Caraveo-Anduaga JJ, Colmenares-Bermúdez E. Los trastornos psiquiátricos y el abuso de sustancias en México: Panorama epidemiológico. Salud Mental 2002;25(2):9-15

23. Díaz-Martínez LR, Campillo Serrano C, Cerrud-Sánchez J, RosadoFranco A et al. El consumo de alcohol en la población psiquiátrica. Anales. Instituto Mexicano de Psiquiatría, Reseña de la VII Reunión de Investigación: 1992;108-116.

24. Díaz-Martínez LR. Comorbilidad psiquiátrica: consumo excesivo de alcohol y trastornos psiquiátricos. Las Adicciones: hacia un enfoque multidisciplinario. México: Secretaría de Salud; 1994.

25. Marín-Navarrete R, Eliosa-Hernández A, Lozano-Verduzco I, Fernández-De la Fuente $\mathrm{C}$ et al. Estudio sobre la experiencia de hombres atendidos en centros residenciales de ayuda mutua para la atención de las adicciones. Salud Mental 2013;36(59):393-402

26. Pulido-Rull MA, Moyers-González M, Martínez-Salas A. Algunos datos acerca del funcionamiento de una muestra de grupos de autoayuda en México. Revista Mexicana de Investigación en Psicología, 2009;1(1):85-97.

27. American Psychiatric Association.APA. DSM-IV-TR: Diagnostic and Statistical Manual of Mental Disorders. Washington, DC. Cuarta edición revisada; 2000.

28. Apiquian R, Páez F, Tapia RO, Fresán A. Validez y confiabilidad de la Escala para la Evaluación de la Manía. Salud Mental 1997;20(3):23-29.

29. Colom F, Vieta E, Martínez-Arán A, García-García M et al. 2002; Versión española de una escala de evaluación de la manía: validez y fiabilidad de la escala de Young. Med Clin Barcelona 1997;119:366-371.

30. Young RC, Biggs JT, Ziegler VE, Meyer DA. A rating scale for mania: Reliability, validity and sensitivity. Br J Psychiatr 1978;133:429-435.

31. Becerra B, Ortega-Soto HA, Torner C. Validez y reproductibilidad del examen cognoscitivo breve (Mini-mental State Examination) en una unidad de cuidados especiales de un hospital psiquiátrico. Salud Mental 1992;15(4):41-45.

32. Folstein MF, Folstein SE, Mc Hugh PR et al. 1975; Minimental state: a practical guide for grading the cognitive state of patients for the clinician. J Psychiatric Res 1992;12:189-198.

33. Kessler y Üstün, 2004; http://www.hcp.med.harvard.edu/wmh/

34. Haro JM, Arbabzadeh-Bouchez S, Brugha TS, De Girolamo G et al. Concordance of the Composite International Diagnostic Interview Version 3.0 (CIDI 3.0) with standardized clinical assessments in the WHO World Mental Health Surveys. International Jf Methods Psychiatric Research 2006;15:167-180.

35. Medina-Mora ME, Borges G, Lara C, Benjet C et al. Prevalence, service use, and demographic correlates of 12-month DSM-IV psychiatric disorders in Mexico: results from the Mexican National Comorbidity Survey. Psychological Medicine 2005;35:1-11.

Artículo sin conflicto de intereses 